\title{
Dynamics of Soluble Programmed Death-Ligand 1 (sPDL1) during Chemotherapy and Its Prognostic Implications in Cancer Patients: Biomarker Development in Immuno-oncology
}

\author{
Hyerim $\mathrm{Ha}, \mathrm{MD}^{1}$ \\ Ju-Hee Bang, MS² \\ Ah-Rong Nam, MS 2 \\ Ji-Eun Park, MS² \\ Mei Hua Jin, MS² \\ Yung-Jue Bang, MD, PhD',2 \\ Do-Youn Oh, MD, PhD',2
}

${ }^{1}$ Department of Internal Medicine,

${ }^{2}$ Cancer Research Institute, Seoul National

University College of

Medicine, Seoul, Korea
Correspondence: Do-Youn Oh, MD, PhD

Department of Internal Medicine,

Seoul National University College of Medicine,

101 Daehak-ro, Jongno-gu, Seoul 03080, Korea

Tel: 82-2-2072-0701

Fax: 82-2-762-9662

E-mail: ohdoyoun@snu.ac.kr

Received May 28, 2018

Accepted September 24, 2018

Published Online October 5, 2018

*This study was partly presented at the 2017

Annual Meeting of ASCO (American Society of

Clinical Oncology).

\begin{abstract}
Purpose
The soluble programmed death-ligand 1 (SPDL1) has immunosuppressive activity and is a candidate biomarker for immuno-oncology drug development. In this study, we measured SPDL1 at pre- and post-chemotherapy and at disease progression to uncover the dynamics of SPDL1 during treatment in biliary tract cancer (BTC) patients.
\end{abstract}

\section{Materials and Methods}

From 90 BTC patients (training cohort, 53; validation cohort, 37) who were candidates for palliative first-line chemotherapy, blood was collected at pre- and post-chemotherapy (at the time of best response) and at disease progression. The SPDL1 levels were measured using an enzyme-linked immunosorbent assay. Responses to chemotherapy, overall survival (OS), and other prognostic factors including the neutrophil-lymphocyte ratio (NLR) were analyzed.

\section{Results}

The OS of all patients was 11.5 months (confidence interval [Cl], 9.7 to 16.2). The best response was complete response in seven (7.8\%), partial response in 20 (22.2\%), stable disease in $52(57.8 \%)$, and disease progression (PD) in 11 patients (12.2\%). Patients with high pre-chemotherapy SPDL1 $(\geq 1.30 \mathrm{ng} / \mathrm{mL}$ ) showed worse OS than patients with low prechemotherapy sPDL1 (9.1 months vs. 12.5 months, $p=0.003$ ). In multivariate analyses, high pre-chemotherapy sPDL1 (hazard ratio [HR], $1.96 ; 95 \% \mathrm{Cl}, 1.2$ to $3.9 ; \mathrm{p}=0.011$ ) and high pre-chemotherapy NLR (HR, 1.82; 95\% $\mathrm{Cl}, 1.1$ to 3.0; $\mathrm{p}=0.020$ ) were independent poor prognostic factors for OS. At the time of PD, SPDL1 was increased significantly compared with pre-chemotherapy sPDL1 (1.59 ng/mL vs. $0.72 \mathrm{ng} / \mathrm{mL}, \mathrm{p}=0.003)$.

\section{Conclusion}

The SPDL1 at pre-chemotherapy confers the prognostic value for OS in BTC patients under palliative chemotherapy. The dynamics of SPDL1 during chemotherapy correlate with disease burden and have prognostic value.

\section{Introduction}

Immunotherapy is an emerging strategy for the treatment of patients with diverse ranges of malignancy. As the concept of cancer immunity is investigated, host immune systems that interact with tumor cells and the microenvironment have been targeted for oncology drug development [1-3]. In biliary tract cancer (BTC), the development of new drugs, including immunological agents, is urgently needed because
Key words

Soluble PDL1, PDL1, Dynamics, Biomarkers, Prognosis
BTC patients have poor prognosis with no effective treatment except a limited number cytotoxic agents.

Programmed death 1 (PD-1) is a key checkpoint and is expressed in activated T cells. Its ligand, programmed deathligand 1 (PD-L1), is induced by active T cells to evade host immune systems. It has been suggested to play a pivotal role in cancer development. PD-1 or PD-L1 protein expression levels in tumor infiltrating lymphocytes and tumor cells serve as candidate biomarkers to predict the prognosis of cancer patients [4-8]. Immune cell infiltration into the 
microenvironment of BTCs is also a prognostic factor [9-11]. The upregulation of PD-L1 and PD-1 in tumor tissues is also reported in BTC [12]. Although many new drugs targeting PD-L1 and PD-1 have been developed, the biomarkers that could predict the efficacy are unknown. The expression of PD-L1 in tumor tissues is one of the candidate biomarkers for the use of anti-PD-L1 or PD-1 antibodies. However, biopsy of tumor tissue is often risky and it is difficult to obtain sufficient amounts of tissue for biomarker analysis. The immune response is not fixed one; it changes across time sequence or is influenced by the ongoing treatment. It is therefore necessary to repeatedly perform biopsies, which is difficult in the real field of clinical practice. In this regard, studies of soluble biomarkers or liquid biopsies are being highlighted. The soluble programmed death-ligand 1 (sPDL1) has immunosuppressive activity and is under investigation as a candidate biomarker for immuno-oncology drug development. In our previous study, we showed that sPDL1 could be used as a biomarker with prognostic value in cancer patients [13].

The purpose of this study was to investigate the changes of sPDL1 levels during chemotherapy and to analyze the relationship of the sPDL1 changes with clinical outcomes. In this study, we measured the serum SPDL1 at pre- and postchemotherapy and at disease progression and evaluated its dynamics and prognostic implications in BTC patients.

\section{Materials and Methods}

\section{Patients and data collection}

Patients with pathologically confirmed recurrent or initially unresectable BTC, who were scheduled to receive palliative chemotherapy were enrolled. Intrahepatic cholangiocarcinoma (IHCC), extrahepatic cholangiocarcinoma (EHCC), gallbladder cancer (GB ca), and ampulla of Vater cancer (AoV ca) were all included.

Ninety patients were recruited at the Seoul National University Hospital. The training cohort included patients treated with S-1/ cisplatin chemotherapy under a phase II clinical trial enrolled between March 2001 and November 2008. The validation cohort included patients treated with gemcitabine/ cisplatin chemotherapy enrolled between October 2008 and February 2016. The training and validation cohorts consisted of 53 and 37 patients, respectively. The blood samples were collected before chemotherapy (prechemotherapy) and at the time of best response (post-chemotherapy) and disease progression.

Demographic and laboratory data, including total biliru- bin, albumin, carcinoembryonic antigen (CEA), carrbohydrate antigen 19-9, and blood cell counts before initiation of chemotherapy, were collected. Neutrophil-to-lymphocyte ratio (NLR, neutrophil/lymphocyte), platelet-to-lymphocyte ratio (PLR, platelet/lymphocyte) and systemic inflammatory index (SII, neutrophil ×platelet/lymphocyte) were calculated. The sPDL1 level was measured using an enzyme-linked immunosorbent assay (ELISA) (PDCD1LG1 ELISA kit, USCN Life Science, Shandong Sheng, TX) in patients' blood samples according to the manufacturer's instructions [13,14]. ELISA is the method to identify peptides, proteins, antibodies and hormones. It is accomplished by assessing the conjugated enzyme activity via incubation with a substrate to detect a measureable sPDL1. We use patient's plasma that was collected from patient's blood by Ficoll-Paque PLUS (GE Healthcare Life Sciences, Uppsala, Sweden). It was kept at $-80^{\circ} \mathrm{C}$ and incubated 1 hour at $37^{\circ} \mathrm{C}$ at ELISA kit well. It was aspirated and washed 3 to 5 times and incubated again. After that procedure, it read at $450 \mathrm{~nm}$ immediately. Each sample was analyzed in duplicate.

The response to chemotherapy was determined according to Response Evaluation Criteria in Solid Tumor (RECIST) ver. 1.1. Overall survival (OS) was calculated from the date of diagnosis for BTC to the date of death or last follow-up date. Progression-free survival (PFS) was calculated from the date of diagnosis to the date of disease progression.

\section{Statistical analysis}

Pearson's chi-square test or Fisher exact test was used for categorical variables, as appropriate. A t test was used for comparison of means. To compare the median values of biomarkers, the Mann-Whitney test was used. Median OS or median PFS was calculated using the Kaplan-Meier method and comparisons of differences between groups were assessed using log-rank tests. Univariate and multivariate analyses for OS were performed using Cox regression models. Factors with $p<0.05$ in univariate analysis were collected and analyzed in multivariate regression models. All statistical tests were two-sided, with significance defined as $\mathrm{p}<$ 0.05. Cut-off values of each variable (sPDL1, NLR, PLR, and SII) for OS predictions were determined by using a minimum p-value approach [15].

\section{Ethical statement}

The study protocol was reviewed and approved by the Institutional Review Board of Seoul National University Hospital (H-1408-039-600). Patients gave informed consent for collecting blood samples to analyze biomarkers. All studies were conducted according to guidelines for biomedical research (Declaration of Helsinki). 


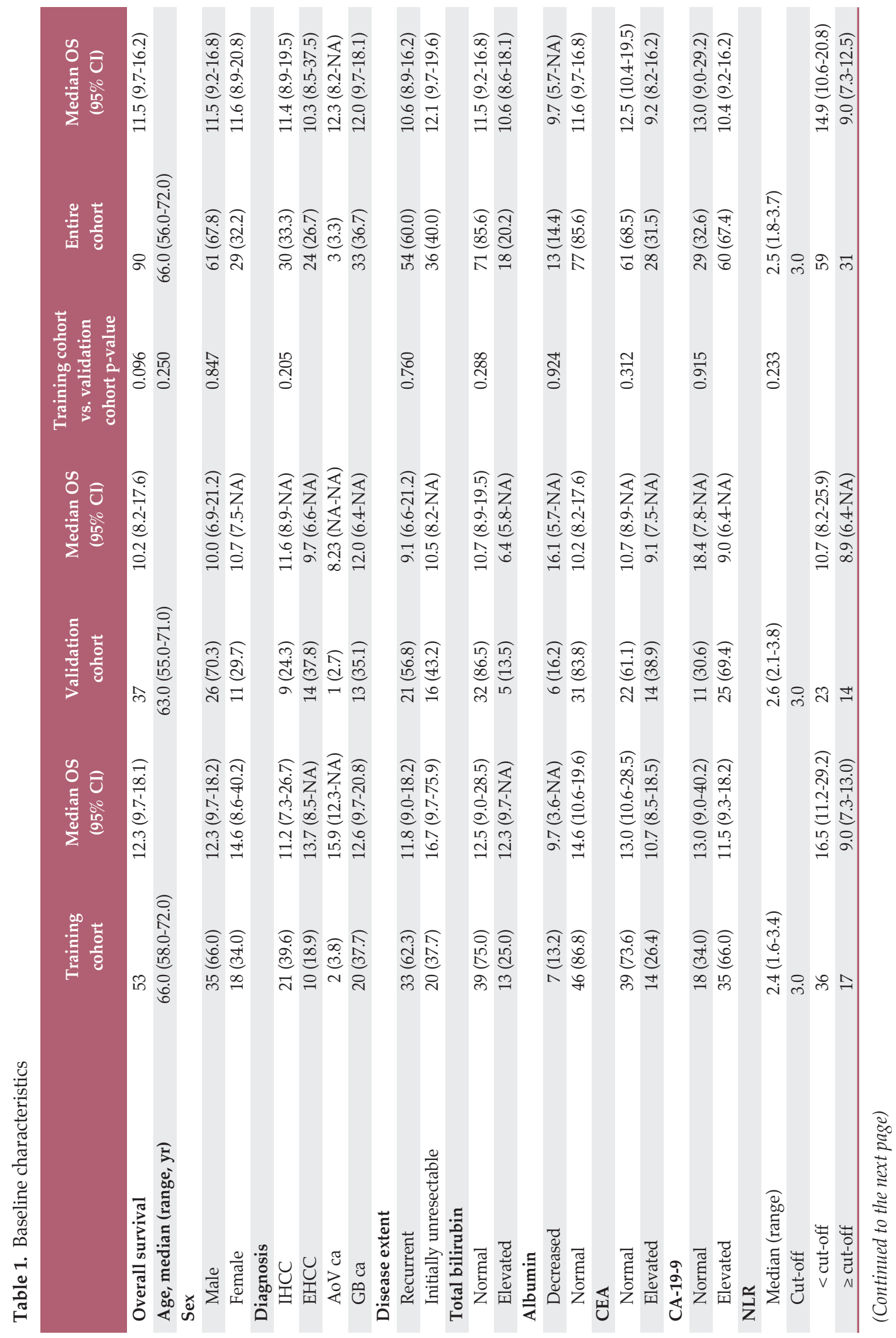




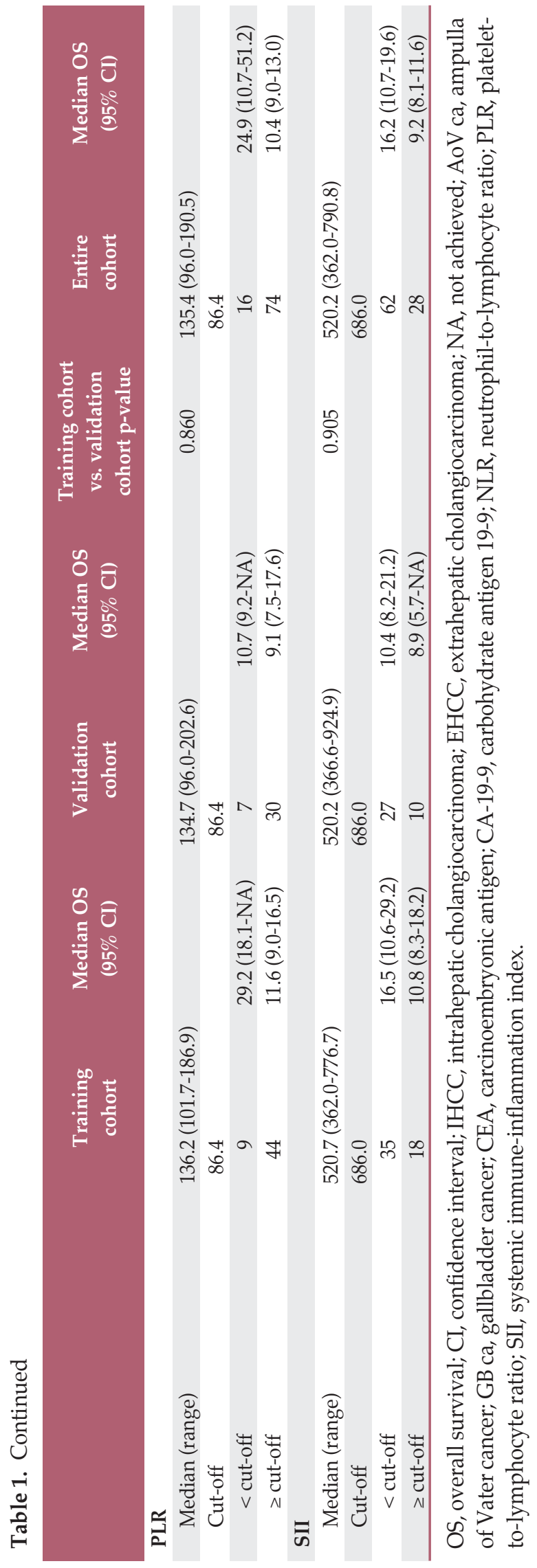

\section{Results}

\section{Patient characteristics}

The median age of the entire cohort was 66-years-old (range, 56.0 to 72.0 years) and the primary sites of cancer were: GB ca, 33 cases (36.7\%); IHCC, 30 cases (33.3\%); EHCC, 24 cases (26.7\%); and AoV ca, three cases (3.3\%). The median OS was 11.5 months (95\% CI, 9.7 to 16.2). Baseline demographic and clinical characteristics were similar between the training and validation cohorts (Table 1). Median values of NLR, PLR, and SII showed no significant differences between the training and validation cohorts. Patients in the training cohort received S-1/ cisplatin as the first-line chemotherapy under clinical trial and patients in the validation cohort received gemcitabine/ cisplatin combination chemotherapy.

\section{Prognostic value of sPDL1}

The median baseline (at pre-chemotherapy) sPDL1 was 0.97 $\mathrm{ng} / \mathrm{mL}$ (range, 0.56 to $1.85 \mathrm{ng} / \mathrm{mL}$ ) and mean was 1.28 (standard deviation, 0.93 ) (Table 2). The cut-off value of sPDL1 for prediction of OS was $1.30 \mathrm{ng} / \mathrm{mL}$ in the training, validation, and entire cohorts (Table 3). The patients with lower baseline sPDL1 showed a better OS (Fig. 1).

When compared with traditional cancer markers and PDL1, high CEA was observed in high sPDL1 (Table 4). It showed statistically significant difference ( $p=0.029$ ).

In univariate analysis for prognostic factors, disease extent (initially unresectable vs. recurrent), number of metastasized organs, NLR, and sPDL1 were significant prognostic factors for OS. In multivariate analysis, sPDL1 and NLR were significant for OS (Table 5).

\section{Dynamics of soluble PD-L1}

The best response was complete response in seven patients, partial response (PR) in 20 patients, stable disease (SD) in 52 patients, and disease progression in 11 patients. At the time of disease progression, sPDL1 was elevated compared with the pre-chemotherapy cases. There was a trend of SPDL1 to decrease when the tumor burden decrease and a trend to increase when the tumor burden increase in each response groups.

In the PR group, sPDL1 levels at pre-chemotherapy and post-chemotherapy were $1.19 \mathrm{ng} / \mathrm{mL}$ and $0.98 \mathrm{ng} / \mathrm{mL}$, respectively. Three samples were collected at disease progression after PR, and the median sPDL1 was $2.77 \mathrm{ng} / \mathrm{mL}$, which was higher than that of the pre-chemotherapy or post-chemotherapy. Similarly, in the SD group, the median sPDL1 at disease 
Table 2. Baseline sPDL1

\begin{tabular}{|c|c|c|c|c|}
\hline & \multicolumn{4}{|c|}{ sPDL1 (ng/mL) } \\
\hline & $\begin{array}{l}\text { Training } \\
\text { cohort } \\
(n=53)\end{array}$ & $\begin{array}{l}\text { Validation } \\
\text { cohort } \\
(\mathrm{n}=37)\end{array}$ & $\begin{array}{l}\text { Training cohort } \\
\text { vs. validation } \\
\text { cohort p-value }\end{array}$ & $\begin{array}{l}\text { Entire } \\
\text { cohort } \\
(\mathrm{n}=90)\end{array}$ \\
\hline Median (range) & $1.01(0.56-2.07)$ & $0.92(0.56-1.48)$ & 0.463 & $0.97(0.56-1.85)$ \\
\hline Mean \pm SD & $1.39 \pm 1.03$ & $1.15 \pm 0.75$ & 0.240 & $1.28 \pm 0.93$ \\
\hline Cut-off & 1.30 & 1.30 & & 1.30 \\
\hline$<$ cut-off & 30 & 26 & & 56 \\
\hline Median OS (95\% CI) & $16.7(12.5-37.5)$ & $12.0(9.2-21.2)$ & & $16.2(10.7-19.6)$ \\
\hline$\geq$ cut-off & 23 & 11 & & 34 \\
\hline Median OS (95\% CI) & $10.6(9.0-16.5)$ & 6.9 (5.8-NA) & & $9.2(8.1-11.6)$ \\
\hline \multicolumn{5}{|c|}{ Disease extent, median (range) } \\
\hline Recurrent & $1.44(0.76-2.24)$ & $1.16(0.72-1.85)$ & 0.399 & $1.35(0.72-2.16)$ \\
\hline Initially unresectable & $0.73(0.41-1.07)$ & $0.71(0.54-1.11)$ & & $0.71(0.47-1.11)$ \\
\hline \multicolumn{5}{|l|}{ Diagnosis, median (range) } \\
\hline IHCC & $1.34(0.56-2.59)$ & $1.10(0.82-1.59)$ & 0.483 & $1.20(0.56-1.85)$ \\
\hline EHCC & $0.77(0.48-1.03)$ & $0.89(0.55-1.21)$ & & $0.89(0.51-1.16)$ \\
\hline AoV ca & $1.68(0.94-2.41)$ & $0.71(0.71-0.71)$ & & $0.94(0.82-1.68)$ \\
\hline GB ca & $1.23(0.54-2.12)$ & $1.10(0.56-1.99)$ & & $1.11(0.56-2.07)$ \\
\hline
\end{tabular}

sPDL1, soluble form programmed death-ligand 1; SD, standard deviation; OS, overall survival; CI, confidence interval; IHCC, intrahepatic cholangiocarcinoma; EHCC, extrahepatic cholangiocarcinoma; AoV ca, ampulla of vater cancer; GB ca, gallbladder cancer.

Table 3. Dynamics of sPDL1

\begin{tabular}{|c|c|c|c|c|c|}
\hline Best response & $\mathrm{CR}(\mathrm{n}=7)$ & PR $(n=20)$ & $\operatorname{SD}(n=52)$ & PD $(n=11)$ & Total $(n=90)$ \\
\hline \multicolumn{6}{|l|}{ Pre-chemo sPDL1 } \\
\hline Median (range) & $0.88(0.49-0.92)$ & $1.19(0.53-1.97)$ & $1.16(0.62-2.20)$ & $0.62(0.35-0.86)$ & $0.97(0.56-1.85)$ \\
\hline Mean \pm standard deviation & $0.73 \pm 0.27$ & $1.42 \pm 0.99$ & $1.44 \pm 0.97$ & $0.67 \pm 0.37$ & $1.28 \pm 0.93$ \\
\hline \multicolumn{6}{|l|}{ Post-chemo sPDL1 } \\
\hline Median (range) & $1.02(0.56-1.28)$ & $0.98(0.69-1.54)$ & $1.19(0.66-1.98)$ & $1.04(0.76-2.86)$ & $1.07(0.66-1.83)$ \\
\hline Mean \pm standard deviation & $1.01 \pm 0.51$ & $1.18 \pm 0.72$ & $1.38 \pm 0.87$ & $1.62 \pm 1.26$ & $1.34 \pm 0.88$ \\
\hline At PD & $\mathrm{n}=0$ & $\mathrm{n}=3$ & $\mathrm{n}=15$ & $\mathrm{n}=11$ & $\mathrm{n}=29$ \\
\hline Median (range) & & $2.77(1.85-2.92)$ & $1.83(0.99-2.41)$ & $1.04(0.76-2.86)$ & $1.59(0.94-2.60)$ \\
\hline Mean \pm standard deviation & & $2.26 \pm 1.15$ & $1.74 \pm 0.94$ & $1.62 \pm 1.26$ & $1.75 \pm 1.07$ \\
\hline
\end{tabular}

sPDL1, soluble form PD-L1; CR, complete remission; PR, partial response; SD, stable disease; PD, progression disease.

progression $(1.83 \mathrm{ng} / \mathrm{mL})$ was higher than that of the prechemotherapy (1.16 ng/mL) and post-chemotherapy (1.19 $\mathrm{ng} / \mathrm{mL}$ ). In the disease progression group, sPDL1 levels at prechemotherapy and disease progression were $0.62 \mathrm{ng} / \mathrm{mL}$ and $1.04 \mathrm{ng} / \mathrm{mL}$, respectively (Table 3, Fig. 2).

\section{Discussion}

In this study, baseline sPDL1 levels (at pre-chemotherapy) predicted the survival of BTC patients treated with palliative chemotherapy, which was comparable to our previous study [13]. High level of sPDL1 had a correlation with worse survival and was shown to be a significant prognostic factor in univariate and multivariate analyses. In terms of dynamic 
A
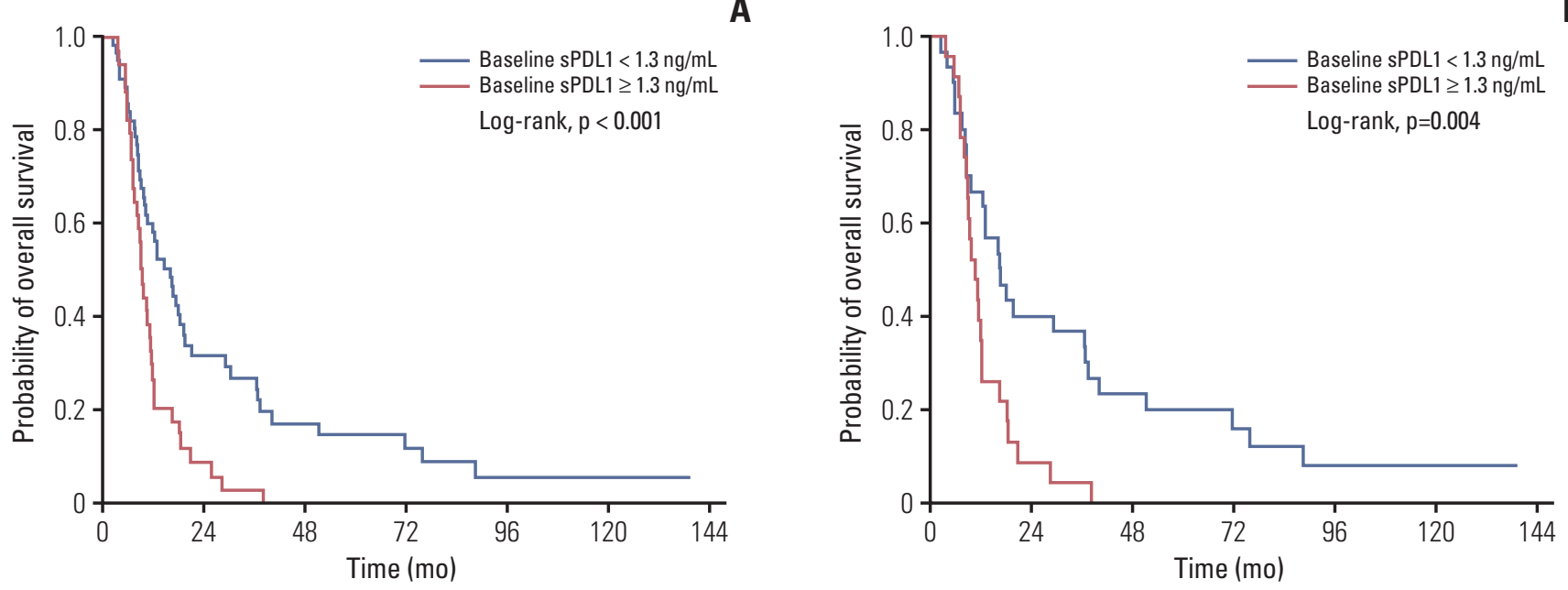

C

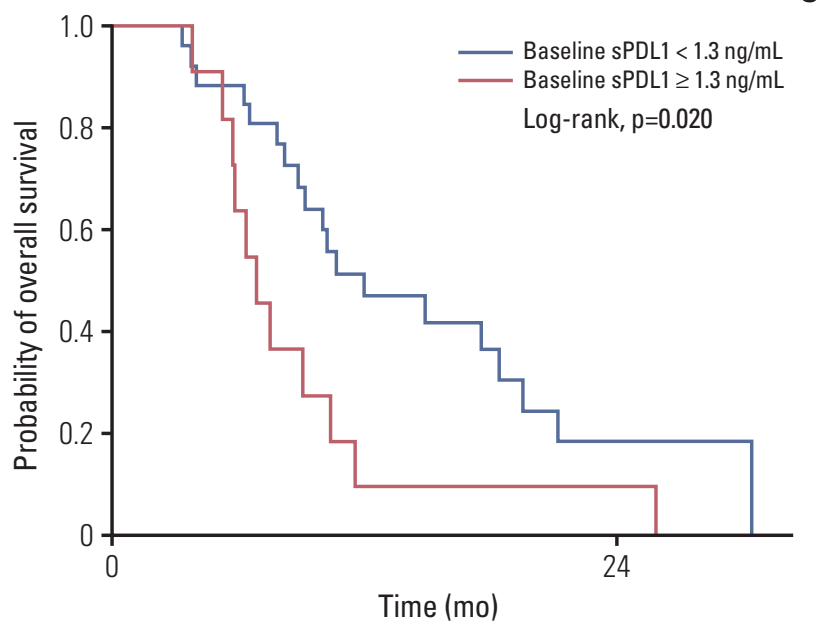

Fig. 1. Overall survival according to pre-chemotherapy soluble programmed death-ligand 1 (sPDL1). Baseline sPDL1 is correlated with overall survival in entire cohort (A), training cohort (B), and validation cohort (C). Cut-off value of sPDL1 is $1.30 \mathrm{ng} / \mathrm{mL}$ in three cohorts. High sPDL1 at pre-chemotherapy confers worse overall survival.

Table 4. Traditional tumor marker (CEA) and sPDL1

\begin{tabular}{|c|c|c|c|c|}
\hline & \multicolumn{2}{|c|}{ sPDL1 (cut-off value 1.3) } & \multirow{2}{*}{ Total } & \multirow{2}{*}{ p-value } \\
\hline & $<1.3$ & $\geq 1.3$ & & \\
\hline \multicolumn{5}{|c|}{ CEA (normal 0-5 ng/mL) } \\
\hline$<10$ & 47 & 21 & 68 & 0.029 \\
\hline$\geq 10$ & 9 & 12 & 21 & \\
\hline Total & 56 & 33 & 89 & \\
\hline
\end{tabular}

CEA, carcinoembryonic antigen; sPDL1, soluble form programmed death-ligand 1. 
Table 5. Prognostic factors for overall survival

\begin{tabular}{|c|c|c|c|c|}
\hline & \multicolumn{2}{|c|}{ Univariate analysis } & \multicolumn{2}{|c|}{ Multivariate analysis } \\
\hline & HR $(95 \% \mathrm{CI})$ & p-value & HR $(95 \%$ CI $)$ & p-value \\
\hline Age & $0.979(0.63-1.52)$ & 0.925 & - & - \\
\hline Diagnosis & $0.976(0.77-1.23)$ & 0.839 & - & - \\
\hline Disease extent & $0.567(0.35-0.92)$ & 0.022 & $0.874(0.50-1.52)$ & 0.636 \\
\hline No. of metastasis organs & $1.842(1.06-3.21)$ & 0.031 & $1.351(0.75-2.45)$ & 0.320 \\
\hline Total bilirubin & $1.275(0.74-2.20)$ & 0.380 & - & - \\
\hline Albumin & $0.553(0.29-1.06)$ & 0.074 & - & - \\
\hline CEA & $1.491(0.91-2.43)$ & 0.110 & - & - \\
\hline CA 19-9 & $1.389(0.86-2.24)$ & 0.177 & - & - \\
\hline sPDL1 & $2.230(1.39-3.55)$ & $<0.001$ & $1.959(1.17-3.29)$ & 0.011 \\
\hline NLR & $2.113(1.31-3.41)$ & 0.002 & $1.823(1.10-3.02)$ & 0.020 \\
\hline PLR & $1.596(0.90-2.82)$ & 0.108 & - & - \\
\hline SII & $1.432(0.89-2.31)$ & 0.141 & - & - \\
\hline
\end{tabular}

HR, hazard ratio; CI, confidence interval; CEA, carcinoembryonic antigen; CA 19-9, carbohydrate antigen 19-9; sPDL1, soluble form of PD-L1; NLR, neutrophil-to-lymphocyte ratio; PLR, platelet-to-lymphocyte ratio; SII, systemic immune-inflammation index.

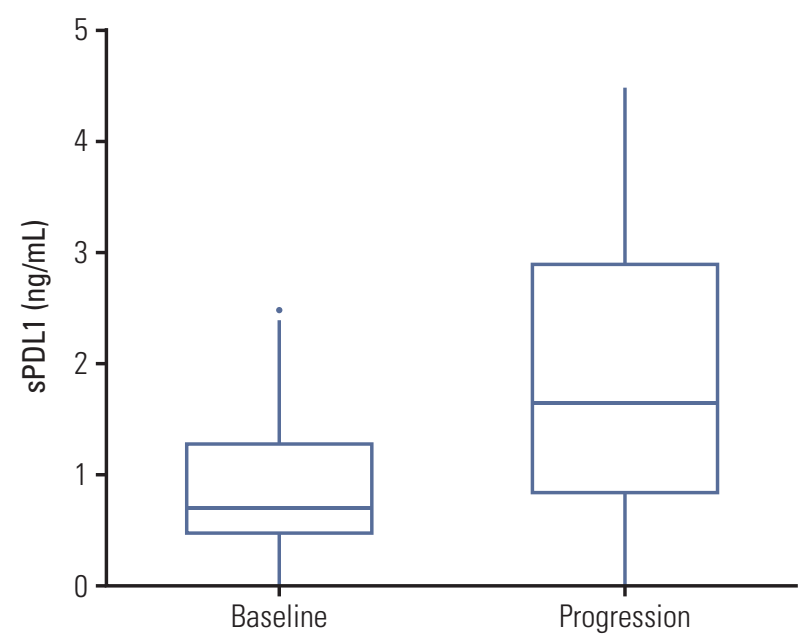

Fig. 2. Changes of soluble programmed death-ligand 1 (sPDL1) at disease progression. sPDL1 level at the time of disease progression is increased compared with pre-chemotherapy.

changes of sPDL1 levels during chemotherapy, the levels were elevated at the time of disease progression compared to that of the pre-chemotherapy.

Tumor cells evade the host immune system by expressing PD-L1, which binds to PD-1 on the surface of activated T or $\mathrm{B}$ cells. PD-1/PD-L1 binding causes inhibitory signaling in T and $\mathrm{B}$ cells, causing suppression of T-cell migration, prolif- eration, and secretion of cytotoxic mediators [1,16-18]. Higher PD-L1 levels in tumor tissues were reported to have a strong correlation with adverse clinical outcomes [19]. Elevated serum sPDL1 is also related with less differentiated tumors, higher invasiveness, and greater metastatic potential in several solid tumors $[20,21]$. We previously reported that high sPDL1 was related with worse survival in BTC patients who received palliative chemotherapy. In this study, high level of sPDL1 is reproducibly correlated with worse survival of BTC patients.

Another important purpose of this study was to uncover the dynamics of sPDL1 during chemotherapy and to determine whether there was a correlation of dynamics of sPDL1 with tumor response or tumor burden. We measured sPDL1 at the time of best response and at the time of disease progression besides pre-chemotherapy. There was a trend of sPDL1 to decrease when the tumor burden decrease and a trend to increase when the tumor burden increase in each response groups. At the time of disease progression, sPDL1 was consistently elevated compared with that of pre-chemotherapy.

There have been few studies regarding the dynamics of expression of PD-L1 in tumor tissues. In subgroup analyses of clinical trials using anti-PD-L1 antibody, the level of tumor tissue PD-L1 protein had a predictive value for the response to the PD-L1 inhibitor in several types of cancer [22]. Another study showed that PD-L1 expression in tumor cells increased in non-small cell lung cancer patients treated with gefitinib [23]. In esophageal cancer patients, the PD-L1 level of tumor cells also increased after neoadjuvant concurrent chemora- 
diotherapy [24]. However, thus far, changes of sPDL1 after treatment have not been reported. Our study is the first report on the dynamics of sPDL1 during chemotherapy.

Our study had limitations. It was a single center study, and a relatively small number of patients. However, this study has value as the first study to uncover the dynamics of sPDL1 in cancer patients treated with palliative chemotherapy. The sPDL1 level was increased noticeably at disease progression. Our finding supports further studies to determine whether sPDL1 could serve as biomarker for immunotherapy. Further study on correlation between sPDL1 and PDL1 expression in tumor tissue is also worthy and needed.

In conclusion, the sPDL1 level at pre-chemotherapy had prognostic value for OS in BTC patients under palliative chemotherapy. The dynamics of sPDL1 during chemotherapy correlated with disease burden and had prognostic value.

\section{Conflicts of Interest}

Conflict of interest relevant to this article was not reported.

\section{Acknowledgments}

This study was supported by 3rd Astrazeneca-KHIDI (Korea Health Industry Development Institute) Oncology Research Program (Grant No. 06-2016-2920), and supported by grant from SNUH Research Fund (Grant No. 03-2017-0100).

\section{References}

1. Pardoll DM. The blockade of immune checkpoints in cancer immunotherapy. Nat Rev Cancer. 2012;12:252-64.

2. Sharma P, Allison JP. Immune checkpoint targeting in cancer therapy: toward combination strategies with curative potential. Cell. 2015;161:205-14.

3. Postow MA, Callahan MK, Wolchok JD. Immune checkpoint blockade in cancer therapy. J Clin Oncol. 2015;33:1974-82.

4. Le DT, Uram JN, Wang H, Bartlett BR, Kemberling H, Eyring $\mathrm{AD}$, et al. PD-1 blockade in tumors with mismatch-repair deficiency. N Engl J Med. 2015;372:2509-20.

5. Chen J, Jiang CC, Jin L, Zhang XD. Regulation of PD-L1: a novel role of pro-survival signalling in cancer. Ann Oncol. 2016;27:409-16.

6. Chen DS, Irving BA, Hodi FS. Molecular pathways: next-generation immunotherapy: inhibiting programmed death-ligand 1 and programmed death-1. Clin Cancer Res. 2012;18:6580-7.

7. Brahmer JR, Drake CG, Wollner I, Powderly JD, Picus J, Sharfman WH, et al. Phase I study of single-agent anti-programmed death-1 (MDX-1106) in refractory solid tumors: safety, clinical activity, pharmacodynamics, and immunologic correlates. J Clin Oncol. 2010;28:3167-75.

8. Nomi T, Sho M, Akahori T, Hamada K, Kubo A, Kanehiro H, et al. Clinical significance and therapeutic potential of the programmed death-1 ligand/programmed death-1 pathway in human pancreatic cancer. Clin Cancer Res. 2007;13:2151-7.

9. Sasada T, Suekane S. Variation of tumor-infiltrating lymphocytes in human cancers: controversy on clinical significance. Immunotherapy. 2011;3:1235-51.

10. Nakakubo Y, Miyamoto M, Cho Y, Hida Y, Oshikiri T, Suzuoki M, et al. Clinical significance of immune cell infiltration within gallbladder cancer. Br J Cancer. 2003;89:1736-42.

11. Goeppert B, Frauenschuh L, Zucknick M, Stenzinger A, Andrulis M, Klauschen F, et al. Prognostic impact of tumourinfiltrating immune cells on biliary tract cancer. Br J Cancer.
2013;109:2665-74.

12. Chai Y. Immunotherapy of biliary tract cancer. Tumour Biol. 2016;37:2817-21.

13. Ha H, Nam AR, Bang JH, Park JE, Kim TY, Lee KH, et al. Soluble programmed death-ligand 1 (sPDL1) and neutrophilto-lymphocyte ratio (NLR) predicts survival in advanced biliary tract cancer patients treated with palliative chemotherapy. Oncotarget. 2016;7:76604-12.

14. Frigola X, Inman BA, Lohse CM, Krco CJ, Cheville JC, Thompson $\mathrm{RH}$, et al. Identification of a soluble form of $\mathrm{B} 7-\mathrm{H} 1$ that retains immunosuppressive activity and is associated with aggressive renal cell carcinoma. Clin Cancer Res. 2011;17:191523.

15. Williams BA, Mandrekar JN, Mandrekar SJ, Cha SS, Furth AF. Finding optimal cutpoints for continuous covariates with binary and time-to-event outcomes. Technical report series No. 79. Rochester, MN: Mayo Foundation; 2006.

16. Thompson RH, Gillett MD, Cheville JC, Lohse CM, Dong H, Webster WS, et al. Costimulatory B7-H1 in renal cell carcinoma patients: Indicator of tumor aggressiveness and potential therapeutic target. Proc Natl Acad Sci U S A. 2004;101: 17174-9.

17. Park JJ, Omiya R, Matsumura Y, Sakoda Y, Kuramasu A, Augustine MM, et al. B7-H1 / CD80 interaction is required for the induction and maintenance of peripheral T-cell tolerance. Blood. 2010;116:1291-8.

18. Butte MJ, Keir ME, Phamduy TB, Sharpe AH, Freeman GJ. Programmed death-1 ligand 1 interacts specifically with the B7-1 costimulatory molecule to inhibit T cell responses. Immunity. 2007;27:111-22.

19. Krambeck AE, Dong H, Thompson RH, Kuntz SM, Lohse CM, Leibovich BC, et al. Survivin and b7-h1 are collaborative predictors of survival and represent potential therapeutic targets for patients with renal cell carcinoma. Clin Cancer Res. 2007; 
13:1749-56.

20. Choueiri TK, Figueroa DJ, Fay AP, Signoretti S, Liu Y, Gagnon $\mathrm{R}$, et al. Correlation of PD-L1 tumor expression and treatment outcomes in patients with renal cell carcinoma receiving sunitinib or pazopanib: results from COMPARZ, a randomized controlled trial. Clin Cancer Res. 2015;21:1071-7.

21. Fukuda T, Kamai T, Masuda A, Nukui A, Abe H, Arai K, et al. Higher preoperative serum levels of PD-L1 and B7-H4 are associated with invasive and metastatic potential and predictable for poor response to VEGF-targeted therapy and unfavorable prognosis of renal cell carcinoma. Cancer Med. 2016;5:1810-20.

22. Herbst RS, Soria JC, Kowanetz M, Fine GD, Hamid O, Gordon
MS, et al. Predictive correlates of response to the anti-PD-L1 antibody MPDL3280A in cancer patients. Nature. 2014;515: 563-7.

23. Han JJ, Kim DW, Koh J, Keam B, Kim TM, Jeon YK, et al. Change in PD-L1 expression after acquiring resistance to gefitinib in EGFR-mutant non-small-cell lung cancer. Clin Lung Cancer. 2016;17:263-70.e2.

24. Lim SH, Hong M, Ahn S, Choi YL, Kim KM, Oh D, et al. Changes in tumour expression of programmed death-ligand 1 after neoadjuvant concurrent chemoradiotherapy in patients with squamous oesophageal cancer. Eur J Cancer. 2016;52: $1-9$. 